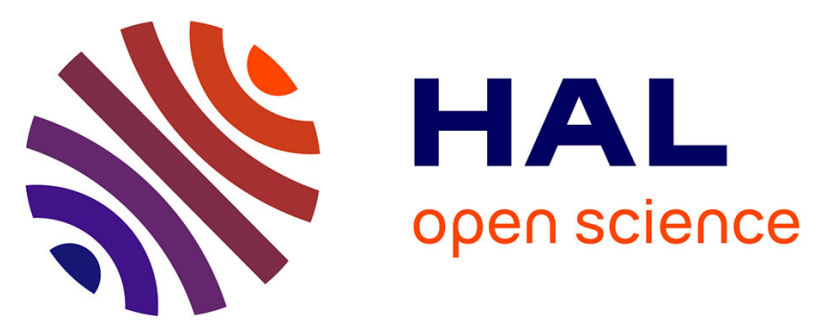

\title{
On Consensus of Double Integrators Over Directed Graphs and with Relative Measurement Bias
}

Srikant Sukumar, Elena Panteley, Antonio Loria, William Pasillas-Lépine

\section{To cite this version:}

Srikant Sukumar, Elena Panteley, Antonio Loria, William Pasillas-Lépine. On Consensus of Double Integrators Over Directed Graphs and with Relative Measurement Bias. 57th IEEE Conference on Decision and Control (CDC 2018), Dec 2018, Miami Beach, FL, United States. pp.4147-4152, 10.1109/CDC.2018.8619329 . hal-02368233

\section{HAL Id: hal-02368233 https://hal.science/hal-02368233}

Submitted on 5 Mar 2020

HAL is a multi-disciplinary open access archive for the deposit and dissemination of scientific research documents, whether they are published or not. The documents may come from teaching and research institutions in France or abroad, or from public or private research centers.
L'archive ouverte pluridisciplinaire HAL, est destinée au dépôt et à la diffusion de documents scientifiques de niveau recherche, publiés ou non, émanant des établissements d'enseignement et de recherche français ou étrangers, des laboratoires publics ou privés. 


\title{
On consensus of double integrators over directed graphs and with relative measurement bias
}

\author{
Srikant Sukumar Elena Panteley Antonio Loría William Pasillas
}

\begin{abstract}
In this paper we investigate sufficient conditions for consensus of double integrators interconnected under constant directed graphs, under the condition that there exists a rooted spanning tree. We assume that only relative position as well as absolute own velocity measurements are available that is, each agent disposes of its own velocity only as well as its position relatively to that of its neighbours. In addition, it is assumed that the relative position measurements are unreliable, in the sense that they are affected by a constant bias. Under these conditions, we provide a consensus algorithm which ensures that the systems stabilize near a common equilibrium point. The analysis is based on Lyapunov direct method and a recent novel approach of analysis of networked systems that takes into account both the synchronization and the collective behavior.
\end{abstract}

\section{INTRODUCTION}

Significant recent strides in analysis of multi-agent consensus protocols have been made in [1], [2], [3], [4] and references therein. Recently a novel framework to analyze synchronization and collective behavior of a network of heterogeneous agents [5]. According with this approach, the complete characterization of the dynamic behavior of networked-interconnected systems coupled via diffusive coupling comprises two essential and dichotomous elements: synchronization and collective behavior. The latter pertains to the dynamics of the networked systems as a whole hence, it is described by what we call emergent dynamics, which may be seen as an "average" of the dynamics of all interconnected systems. In that light, synchronization may be described as the "alignment" of each agent's behavior relative to the emergent dynamics.

Thus, from a dynamical-systems viewpoint, the synchronization problem may be recasted as that of stability analysis of two interconnected dynamical systems evolving in orthogonal spaces. That of the synchronization errors and that of the emergent dynamics. Then, for the purpose of analysis it is assumed in [5] that the emergent dynamics possesses a stable attractor and we say that dynamic consensus is reached if the trajectories of each agent converge asymptotically to those produced by the emergent dynamics.

Even though only the analysis problem for the relative degree one systems with linear coupling is broached in [5], the framework serves as a basis for network control design. Indeed, by modifying the consensus control law, not only may one achieve the synchronization objective but

S. Sukumar is with the Systems and Control Group of the Indian Institute of Technology, Mumbay, India. The other authors are with the CNRS, France. L2S-CentraleSuplec, 91192 Gif-sur-Yvette, France. E-mail: loriallss.supelec.fr. E. Panteley is also with ITMO University, Kronverkskiy av. 49, Saint Petersburg, 197101, Russia. also govern the consensus control goal. This is possible by designing a second control law to govern the emergent dynamics and steer its trajectories to a desired equilibrium.

In the current article, we take a first step forward into the dynamic consensus control problem by addressing it in the context of networks of relative-degree two (driftless) systems interconnected over a directed graph. The motivation stems from mechanical systems with the assumption that only relative position and absolute velocity are available for feedback. However, relative position sensors suffer from errors such as bias in measurements. If not compensated this bias could drive the agents to infinity when using relative position feedback for consensus.

In the continuous systems context, bias uncertainties in measurements are relatively sparsely studied. In the context of a single rigid-body system, 'gyro bias' is the most commonly addressed bias uncertainty and has been studied in detail in several references including [6], [7], [8], [9]. However, the literature on adaptive compensation of position sensor bias even for a single rigid-body is rather limited and the only relevant contribution known to the authors is by [10].

The rest of the paper is organized as follows. In the next section we formulate the problem and recall the essentials of the analysis framework of [5]; hence, we derive the network model in appropriate coordinates. In Section III we present our main results. Some simulation results that illustrate our theoretical findings are presented in Section IV, before concluding with some remarks in Section V.

\section{PROBLEM FORMULATION AND NETWORK MODEL}

We consider the consensus problem for a set of $N$ doubleintegrator systems

$$
\begin{aligned}
& \dot{x}_{1 i}=x_{2 i} \\
& \dot{x}_{2 i}=u_{i}, \quad i \leq N,
\end{aligned}
$$

interconnected over a directed graph. To that effect, we dispose of decentralized controllers $u_{i} \in \mathbb{R}$ with $i \leq N$ which may be designed in function of the available measurements which are relative positions, yet affected by a constant measurement bias, as well as own velocities. More precisely, we do not assume that either $x_{1 i}, x_{2 i}$, or estimates of the latter are individually available for measurement, but rather the quantity

$$
\hat{x}_{1 i}-\hat{x}_{1 j}=x_{1 i}-x_{1 j}+\delta_{i j}
$$

where $\delta_{i j}$ denotes the constant bias. 
Thus, we pose the following consensus control law

$$
u_{i}:=-\sum_{j=1}^{N} \ell_{i j}\left(\hat{x}_{1 i}-\hat{x}_{1 j}\right)-\gamma x_{2 i}+v_{i}
$$

where $v_{i}$ is an additional control input that is to be designed to compensate for the effect of the bias and the disturbance.

Now, to simplify the notation, let us define

$$
v:=\left[\begin{array}{c}
v_{1} \\
\vdots \\
v_{N}
\end{array}\right], \quad \delta:=\left[\begin{array}{c}
\delta_{1} \\
\vdots \\
\delta_{N}
\end{array}\right], \quad \delta_{i}:=\sum_{j=1}^{N} \ell_{i j} \delta_{i j} .
$$

Then, the control law takes the form

$$
u=-L x_{1}-\gamma x_{2}+v-\delta
$$

where the Laplacian matrix $L$ is defined as follows

$$
L_{i j}= \begin{cases}\sum_{k \neq i} l_{i k} & \text { if } j=i \\ -l_{i j} & \text { if } j \neq i\end{cases}
$$

and, therefore, the closed-loop network dynamics becomes

$$
\begin{aligned}
& \dot{x}_{1}=x_{2} \\
& \dot{x}_{2}=-L x_{1}-\gamma x_{2}+v-\delta .
\end{aligned}
$$

The control problem boils down to designing the additional control $v$, which may depend only on biased relative position measurements and local velocities to stabilize the network over a synchronization manifold. The latter may be defined in a number of ways; for instance

$$
\mathcal{S}_{c}:=\left\{x_{1} \in \mathbb{R}^{N}, x_{2} \in \mathbb{R}^{N}: x_{1 i}-x_{1}^{*}=0, x_{2 i}-x_{2}^{*}=0\right\}
$$

meaning that all systems stabilize to a common set point. Such behavior, however, does not imply that the trajectories of each pair of systems are convergent to each other that is, that $x_{1 i} \rightarrow x_{1 j}$ and $x_{2 i} \rightarrow x_{2 j}$. Therefore, one can set as control goal to stabilize the manifold

$$
\begin{aligned}
\mathcal{S}_{e}:=\left\{x_{1} \in \mathbb{R}^{N}, x_{2} \in\right. & \mathbb{R}^{N}, i, j \in[1, N]: \\
& \left.x_{1 i}-x_{1 j}=0, x_{2 i}-x_{2 j}=0\right\} .
\end{aligned}
$$

Nonetheless, focusing on the stabilization of the manifold $\mathcal{S}_{e}$ only leads to the networked systems synchronization without knowledge of the collective behavior of the systems. For instance, one may obtain that the manifold $\mathcal{S}_{e}$ is asymptotically stable but all the systems' states $x_{1}, x_{2}$ grow unbounded.

To understand the complete behavior of the network, one needs to examine both, the collective behavior and the synchronization error dynamics. According with the framework in [5] the former is described by the emergent dynamics, which, roughly speaking, consists in a weighted average of the individual systems dynamics. Therefore, it consists in a dynamical system with its own state, that we shall denote by $\left(s_{1}, s_{2}\right) \in \mathbb{R}^{2}$. Thus the synchronization manifold shall be defined as

$$
\begin{aligned}
\mathcal{S}_{e}:=\left\{x_{1} \in \mathbb{R}^{N}, x_{2} \in\right. & \mathbb{R}^{N}, i, j \in[1, N]: \\
x_{1 i}-s_{1} & \left.=0, x_{2 i}-s_{2}=0\right\} .
\end{aligned}
$$

In the sequel, we proceed to exhibit this inherent dichotomous structure of the networked system (6) thereby, posing the appropriate basis for the solution of the consensus problem of double integrators under measurement bias and measurement bias.

\section{A. A dichotomous network model}

By construction, the Laplacian matrix $L$ shown in (5) has at least one eigenvalue equal to zero, that we shall denote $\lambda_{1}$ while all the others have non-negative real parts. Furthermore, if the network graph has a rooted spanning tree the zero eigen-value $\lambda_{1}$ has algebraic multiplicity equal to one and the others have positive real parts, i.e., $0=$ $\Re e\left\{\lambda_{1}\right\}<\Re e\left\{\lambda_{2}\right\} \leq \cdots \leq \Re e\left\{\lambda_{N}\right\}$. Moreover, the right and left eigenvectors corresponding to $\lambda_{1}$, denoted $\vartheta_{r 1} \in \mathbb{R}^{N}$ and $\vartheta_{\ell 1} \in \mathbb{R}^{N}$, are given by

$$
\vartheta_{r 1}^{\top}=\mathbf{1}_{N}^{\top}:=[1, \ldots, 1], \quad \vartheta_{\ell 1}^{\top}=\left[\nu_{1}, \ldots, \nu_{N}\right]
$$

If, in addition, the graph is strongly connected, $\nu_{i}>0$ for all $i \in \mathcal{I}$-see [11].

Thus, as we shall see the "weighted averaged" states of the emergent dynamics are naturally defined using the left eigen vector $\vartheta_{\ell}$, that is, $s:=\left[\begin{array}{ll}s_{1} & s_{2}\end{array}\right]^{\top}$ is a projection of $x:=\left[\begin{array}{ll}x_{1} & x_{2}\end{array}\right]^{\top}$ on the subspace generated by $\vartheta_{\ell}$.

Now, for the Laplacian $L$ there exists a Jacobian decomposition of the form $L=U \Lambda U^{-1}$ where $U \in \mathbb{C}^{N \times N}$ is nonsingular and $\bar{\Lambda} \in \mathbb{C}^{N \times N}$ is a block-diagonal Jordan matrix

$$
\bar{\Lambda}:=\operatorname{diag}\left[\Lambda_{1}, \ldots, \Lambda_{m}\right] .
$$

For a network with a spanning tree, $\Lambda_{1}$ is actually scalar and equals to zero while $\Lambda_{i}$ are Jordan blocks of appropriate dimensions. For the sequel, we rewrite $\bar{\Lambda}$ as

$$
\bar{\Lambda}:=\operatorname{blockdiag}[0 \Lambda], \quad \Lambda:=\operatorname{blockdiag}\left[\Lambda_{2} \cdots \Lambda_{m}\right] .
$$

Remark 1 It is important to stress, at this point, that even though, in general, the Jordan blocks $\Lambda_{i}$ are complex, they may be replaced by a real upper block diagonal matrix — see [12]. Therefore, in the sequel, without loss of generality we will manipulate the matrix $\Lambda$ in (9) as a real matrix.

Furthermore, the matrix $U$ is composed of generalized right-eigenvectors of $L$ among which the first is $v_{r}=\mathbf{1}_{N}$ hence, for further development, we decompose $U$ as

$$
U=\left[\begin{array}{ll}
\mathbf{1}_{N} & U_{1}
\end{array}\right],
$$

where $U_{1} \in \mathbb{C}^{N \times N-1}$. In this case, the first row of $U^{-1}$ corresponds to the first left eigen-vector of $L, v_{\ell}$. Therefore, we may decompose $U^{-1}$ as

$$
U^{-1}=\left[\begin{array}{c}
\vartheta_{\ell}^{\top} \\
U_{1}^{\dagger}
\end{array}\right]
$$

and, necessarily,

$$
\vartheta_{\ell}^{\top} U_{1}=0
$$

It is also convenient to note that

$$
U_{1}^{\dagger} U_{1}=I_{N-1},
$$


and the Laplacian satisfies

$$
L=U_{1} \Lambda U_{1}^{\dagger}
$$

Based on the latter, it becomes natural to introduce the new network coordinates

$$
\xi:=\left[\begin{array}{l}
\xi_{1} \\
\xi_{2}
\end{array}\right], \quad \begin{array}{lll}
\xi_{1} & =U^{-1} x_{1} \\
\xi_{2} & =U^{-1} x_{2}
\end{array}
$$

so the network dynamics becomes

$$
\begin{aligned}
& \dot{\xi}_{1}=\xi_{2} \\
& \dot{\xi}_{2}=-U^{-1} U \bar{\Lambda} U^{-1} x_{1}-\gamma U^{-1} x_{2}-U^{-1} \delta+U^{-1} v .
\end{aligned}
$$

Now, to compact the notation, we introduce

$$
\theta:=U^{-1} \delta, \quad \hat{\theta}:=U^{-1} v
$$

and $\tilde{\theta}=\hat{\theta}-\theta$. Then,

$$
\begin{aligned}
& \dot{\xi}_{1}=\xi_{2} \\
& \dot{\xi}_{2}=-\bar{\Lambda} \xi_{1}-\gamma \xi_{2}+\tilde{\theta}
\end{aligned}
$$

The system (15) fully describes the dynamic model of the network in the sense that it contains the information regarding both, the synchronization-errors and the consensus dynamics. To see this more clearly, we stress that $\xi_{1}$ and $\xi_{2}$ may, in turn, be subdivided into

$$
\xi_{1}:=\left[\begin{array}{l}
s_{1} \\
\eta_{1}
\end{array}\right], \quad \xi_{2}\left[\begin{array}{l}
s_{2} \\
\eta_{2}
\end{array}\right]
$$

where

$$
s_{i}:=\vartheta_{\ell}^{\top} x_{i}, \quad \text { and } \quad \eta_{i}:=U_{1}^{\dagger} x_{i} .
$$

Thus, the scalar variables $s_{i}$ describe the sate of the averaged dynamics with respect to which the synchronization errors, which are defined as

$$
e:=\left[\begin{array}{l}
e_{1} \\
e_{2}
\end{array}\right]=\left[\begin{array}{l}
x_{1}-\mathbf{1}_{N} s_{1} \\
x_{2}-\mathbf{1}_{N} s_{2}
\end{array}\right] .
$$

Note that $\eta_{i}=0$ if and only if $e_{i}=0$ for $i \in\{1,2\}$. Indeed, since $U_{1}$ is full-column rank, we have

$$
\begin{aligned}
\eta_{i}=0 & \Longleftrightarrow U_{1} \eta_{i}=0 \\
& \Longleftrightarrow\left[I-\mathbf{1}_{N} \vartheta_{\ell}^{\top}\right] x_{i}=0 \\
& \Longleftrightarrow x_{i}-\mathbf{1}_{N} s_{i}=0 .
\end{aligned}
$$

Also, the effect of the perturbations is split into

$$
\theta^{\top}:=\left[\theta_{s}, \theta_{\eta}^{\top}\right]
$$

where

$$
\theta_{s}:=\vartheta_{\ell}^{\top} \delta, \quad \theta_{\eta}:=U_{1}^{\dagger} \delta
$$

are decoupled perturbations: the disturbance $\theta_{s}$ affects the average dynamics

$$
\begin{aligned}
& \dot{s}_{1}=s_{2} \\
& \dot{s}_{2}=-\gamma s_{2}+\tilde{\theta}_{s}, \quad \gamma>0
\end{aligned}
$$

while the disturbance $\theta_{\eta}$, that affects the synchronization error dynamics, which is given by

$$
\dot{\eta}_{1}:=\eta_{2}
$$

$\dot{\eta}_{2}:=-\Lambda \eta_{1}-\gamma \eta_{2}+\tilde{\theta}_{\eta}, \quad$-with $-\Lambda$ Hurwitz.

In addition, in the computation of the latter, we have introduced the additional control input

$$
v:=\mathbf{1}_{N} \hat{\theta}_{s}+U_{1} \hat{\theta}_{\eta}
$$

where $\hat{\theta}_{s}$ and $\hat{\theta}_{\eta}$ are left to be designed to stabilize the respective trivial solutions of the average dynamics (20) and (21). Indeed, consensus is reached if and only if $\eta_{i} \rightarrow 0$ for $i \in\{1,2\}$. Note, however, that this does not imply that consensus is reached to a set point but to the attractor of the mean-field system (20). Hence the interest of also stabilizing (if possible, under the constraints imposed by the lack of absolute and global measurements) the consensus dynamics. In the following section we design the update laws for

$$
\hat{\theta}=\left[\begin{array}{l}
\hat{\theta}_{s} \\
\hat{\theta}_{\eta}
\end{array}\right]=\left[\begin{array}{l}
\vartheta_{\ell}^{\top} v \\
U_{1}^{\dagger} v
\end{array}\right]
$$

and we analyze the stability of both systems (20) and (21).

\section{MAIN RESULTS}

\section{A. Consensus under reliable measurements}

Let us assume, for the time-being, that the disturbance $d \equiv 0$ and the relative measurements are reliable. That is, consider the system (21) with $\tilde{\theta}_{\eta}=0$. We have the following.

Proposition 1 The null solution of (21) with $\tilde{\theta}_{\eta}=0$ is globally exponentially stable.

Proof: The system may be rewritten in the form

$$
\begin{aligned}
& \dot{\eta}=\mathcal{A} \eta, \quad \eta:=\left[\eta_{1}^{\top} \eta_{2}^{\top}\right]^{\top} \\
& \mathcal{A}:=\left[\begin{array}{rc}
0 & I \\
-\Lambda & -\gamma I
\end{array}\right] .
\end{aligned}
$$

The eigenvalues of $\mathcal{A}$ are solutions to the equation (in $\lambda$ )

$$
\begin{aligned}
& \operatorname{det}\left[\begin{array}{cc}
\lambda I & -I \\
\Lambda & (\gamma+\lambda) I
\end{array}\right]=0 \\
\Longleftrightarrow & \operatorname{det}\left[\begin{array}{cc}
\lambda I & -I \\
\Lambda+(\gamma+\lambda) \lambda I & 0
\end{array}\right]=0 \\
\Longleftrightarrow & \operatorname{det}[-\Lambda-(\gamma+\lambda) \lambda I]=0 .
\end{aligned}
$$

Now, since $-\Lambda$ is Hurwitz, its eigenvalues, that we shall denote $\sigma_{i}$, have negative real parts and, according to the last equality above, they satisfy

$$
-\sigma_{i}=(\gamma+\lambda) \lambda \quad \forall i \leq N-1 .
$$

Hence, they are solutions to the equations

$$
\lambda^{2}+\gamma \lambda+\sigma_{i}=0 \quad \gamma>0, \forall i \leq N-1
$$

and have negative real parts for appropriate values if, for each $i \leq N-1$,

$$
\gamma>\frac{2\left[\Re\left\{\sigma_{i}\right\}^{2}+\Im\left\{\sigma_{i}\right\}^{2}\right]}{\Re\left\{\sigma_{i}\right\}}
$$

where $\Re\left\{\sigma_{i}\right\}$ and $\Im\left\{\sigma_{i}\right\}$ represent the real and imaginary parts of $\sigma_{i}$. 
Let us now consider the behavior of the average dynamics (20) with $\tilde{\theta}_{s}=0$ that is,

$$
\left[\begin{array}{c}
\dot{s}_{1} \\
\dot{s}_{2}
\end{array}\right]=\left[\begin{array}{cc}
0 & 1 \\
0 & -\sigma_{s}
\end{array}\right]\left[\begin{array}{l}
s_{1} \\
s_{2}
\end{array}\right] .
$$

We see that $s_{2}(t)=s_{2}(0) \mathrm{e}^{-\gamma t}$ and $s_{1}(t)=s_{1}(0)+$ $\frac{1}{\gamma} s_{2}(0)\left[1-\mathrm{e}^{-\gamma t}\right]$. That is, $s_{2} \rightarrow 0$ and $s_{1}(t) \rightarrow s_{1}(0)+$ $\frac{1}{\gamma} s_{2}(0)$ as $t \rightarrow \infty$ hence, consensus is reached.

The previous developments are summarized in the following statement.

Proposition 2 Consider the systems (1) with $d_{i} \equiv 0$, in closed loop with (3), which uses only relative position measurements as in (2), and let $\delta_{i j}=0$. Let these systems be interconnected over a directed graph containing a rooted spanning tree. Then, the systems achieve set-point consensus if $\gamma>0$.

\section{B. Consensus under unreliable measurements and influence of external disturbances}

Let us consider now the system under the influence of the additive constant disturbance and the measurement bias, (6). Equivalently, we consider the decoupled average system's dynamics (20) and the synchronization error dynamics (21).

To ensure that the collective behavior tends to a steadystate, we need to steer the variable $s_{2}$ in (20b) to zero. This equation is

$$
\dot{s}_{2}=-\gamma s_{2}+\hat{\theta}_{s}-\theta_{s}
$$

where $\theta_{s}$ is constant. Therefore, we see that a simple integral action

$$
\dot{\hat{\theta}}_{s}=-\alpha s_{2}
$$

should compensate for the effect of $\theta$. Note, however, that at first sight the update law requires the measurement of $s_{2}$ which, roughly, corresponds to a weighted average of all neighbors' velocities. Hence, $s_{2}$ may seem unavailable from local measurements. To show that (29) is actually implementable with only local information we define $v_{i}$ in (3) as

$$
\dot{v}_{i}=-\alpha x_{2 i}+\mu_{i}, \quad \alpha>0
$$

where $\mu_{i}$ is to be chosen subsequently. This choice yields for the cumulative multi-agent dynamics,

$$
\dot{v}=-\alpha x_{2}+\mu
$$

where we introduced $v=\left[v_{1} \cdots v_{N}\right]^{\top}$ and $\mu=$ $\left[\mu_{1} \cdots \mu_{N}\right]^{\top}$.

Now, using the transformation proposed in (23), we can now write the parameter update law (30) as

$$
\dot{\hat{\theta}}_{s}=\vartheta_{\ell}^{T} \dot{v}=-\alpha s_{2}+\vartheta^{T} \mu=\dot{\tilde{\theta}}_{s}
$$

so the mean-field system in the presence of disturbance and bias is modified to

$$
\begin{aligned}
& \dot{s}_{1}=s_{2} \\
& \dot{s}_{2}=-\gamma s_{2}+\tilde{\theta}_{s}, \quad \gamma>0 \\
& \dot{\tilde{\theta}}_{s}=-\alpha s_{2}+\vartheta^{T} \mu, \quad \alpha>0 .
\end{aligned}
$$

At this point, we chose $\mu$ to be orthogonal to the space of the average dynamics that is, it must satisfy $\vartheta_{\ell}^{\top} \mu=0$. Indeed, in this case, equations (34), (35) become

$$
\left[\begin{array}{c}
\dot{s}_{2} \\
\dot{\tilde{\theta}}
\end{array}\right]=\left[\begin{array}{ll}
-\gamma & 1 \\
-\alpha & 0
\end{array}\right]\left[\begin{array}{c}
s_{2} \\
\tilde{\theta}
\end{array}\right] .
$$

A direct computation shows that the matrix above is Hurwitz for any positive values of $\alpha$ and $\gamma$.

Let us analyze now the qualitative behavior of the synchronization-error dynamics in the projected coordinates $\left(\eta_{1}, \eta_{2}\right)$, that is, system (21). The task at hand is, as for the mean-field system, to define $\hat{\theta}_{\eta}$ in order to guarantee that $\eta_{2} \rightarrow 0$ and $\eta_{1} \rightarrow$ const. Since the relative measurements are biased, however, it is expected that, the systems reach consensus with a steady state offset. With that in mind let us define the new synchronization error $y:=\Lambda \eta_{1}-\tilde{\theta}_{\eta}$ which, as a matter of fact, is available from the measurements since

$$
y=U_{1}^{\dagger}\left[L x_{1}+\delta\right]-\hat{\theta}_{\eta}
$$

in which $\left[L x_{1}+\delta\right]$ corresponds precisely to the biased relative position measurement and, in view of (23) and (31), $\hat{\theta}_{\eta}$ satisfies

$$
\dot{\hat{\theta}}_{\eta}=U_{1}^{\dagger} \dot{v}=-\alpha \eta_{2}+U_{1}^{\dagger} \mu
$$

Next, consider the function

$$
V\left(y, \eta_{2}\right):=\frac{1}{2}\left[|y|^{2}+\left|\eta_{2}\right|^{2}\right]
$$

whose total derivative along the trajectories of the error dynamics

$$
\begin{aligned}
\dot{y} & =\Lambda \eta_{2}-\dot{\tilde{\theta}}_{\eta} \\
\dot{\eta}_{2} & =-y-\gamma \eta_{2}
\end{aligned}
$$

yields

$$
\dot{V}=\frac{1}{2} y_{\eta}^{\top}\left[\Lambda+\Lambda^{\top}\right] \eta_{2}-y_{\eta}^{\top} \dot{\tilde{\theta}}_{\eta}-\eta_{2}^{\top} y_{\eta}-\gamma\left|\eta_{2}\right|^{2} .
$$

Then, we set

$$
U_{1}^{\dagger} \mu:=\sigma y_{\eta}, \quad \text { with } \quad \sigma>0
$$

or, equivalently, $\mu:=\sigma U_{1} y$-note that this definition satisfies the relation $\vartheta_{\ell}^{\top} \mu=0$. That is, $\mu$ has an influence only in the space of the synchronization errors. Hence, using (41), (37), and $\dot{\tilde{\theta}}=\dot{\hat{\theta}}$, in (40) we obtain

$$
\dot{V} \leq \frac{\lambda_{M}+\alpha+1}{2}\left[\left|y_{\eta}\right|^{2}+\left|\eta_{2}\right|^{2}\right]-\gamma\left|\eta_{2}\right|^{2}-\sigma\left|y_{\eta}\right|^{2} .
$$

where $\lambda_{M}:=\sqrt{\lambda_{\max }\left(\Lambda^{\top} \Lambda\right)}$.

It follows that, provided that

$$
c:=\frac{1}{3} \min \{\gamma, \sigma\} \geq \frac{\lambda_{M}+\alpha+1}{2},
$$

and defining $\zeta:=\left[y_{\eta}^{\top} \eta_{2}^{\top}\right]^{\top}$, we obtain

$$
\dot{V}(\zeta(t)) \leq-2 c V(\zeta(t))
$$

hence,

$$
|\zeta(t)| \leq|\zeta(0)| \mathrm{e}^{-c t}
$$


that is, $\eta_{2} \rightarrow 0$. Furthermore,

$$
\tilde{\theta}_{\eta}(t)=\tilde{\theta}_{\eta}(0)+\int_{0}^{t}\left(\sigma y_{\eta}(\tau)-\alpha \eta_{2}(\tau)\right) d \tau .
$$

Therefore,

$$
\begin{aligned}
\left|\tilde{\theta}_{\eta}(t)\right| & \leq\left|\tilde{\theta}_{\eta}(0)\right|+\int_{0}^{t}\left|(\sigma,-\alpha)^{T}\right||\zeta(\tau)| d \tau \\
& \leq\left|\tilde{\theta}_{\eta}(0)\right|+\sqrt{\sigma^{2}+\alpha^{2}} \int_{0}^{t}|\zeta(\tau)| d \tau \\
& \leq\left|\tilde{\theta}_{\eta}(0)\right|+\frac{\sqrt{\sigma^{2}+\alpha^{2}}}{c}|\zeta(0)|
\end{aligned}
$$

which, in turn, and since $y(t) \rightarrow 0$, implies that

$$
\left|\eta_{1}(t)\right| \leq \frac{1}{\lambda_{m}}\left[\left|\tilde{\theta}_{\eta}(0)\right|+\frac{\sqrt{\sigma^{2}+\alpha^{2}}}{c}|\zeta(0)|\right] .
$$

That is, $\eta_{1}$, or equivalently the synchronization errors, are ultimately bounded by a bound that is inversely proportional to the smallest eigenvalue of the Laplacian. However, increasing $\gamma$ and $\sigma$ affects $c:=\min \{\gamma, \sigma\}$ which satisfies $\sqrt{\sigma^{2}+\alpha^{2}} / c \geq 1$. Therefore, the previous bound is somewhat conservative. On the other hand, this is expected since the part of the perturbation is induced by the measurements.

The previous developments are summarized in the following statement, which is our second main result.

Proposition 3 Consider the systems (1) in closed loop with (3), (2) and

$$
\dot{v}=-\alpha x_{2}+\sigma\left(\left[L x_{1}+\delta\right]-v\right) .
$$

Let the coefficients $\ell_{i j}$ be such that the interconnected systems form a directed graph with a rooted spanning tree. Then, the systems achieve set-point consensus in a practical sense if

$$
\frac{1}{3} \min \{\gamma, \sigma\} \geq \frac{\lambda_{M}+\alpha+1}{2}
$$

where $\lambda_{M}:=\sqrt{\lambda_{\max }\left(\Lambda^{\top} \Lambda\right)}$ and $\Lambda$ corresponds to the block diagonal matrix corresponding to the Jordan decomposition of $L$.

\section{Simulation RESUlts}

Simulations on a test setup of 10 agents connected via a directed graph as shown in figure 1 were carried out to verify the claims made in Propositions 2 and 3.

The initial conditions for the numerical experiments were assumed to be, $x_{1}(0)=[-10,9,2,-4,2,7,8,-6,-4,2]^{T}$, $x_{2}(0)=[0.3,0.2,-0.5,0.4,1,-0.7,0.2,-0.4,-0.2,0.3]^{T}$, and $v(0)=0$.

\section{A. Case of reliable measurements}

We implement Proposition 2 for the case when we have perfect measurements. The parameter, $\gamma=2$ for these simulations. In the absence of bias in measurements, it is evident from figure 2 that the synchronization errors $\left(\eta_{1}(t)\right)$ converge to zero and the average values $\left(s_{1}(t)\right)$ converge to -1.21 .

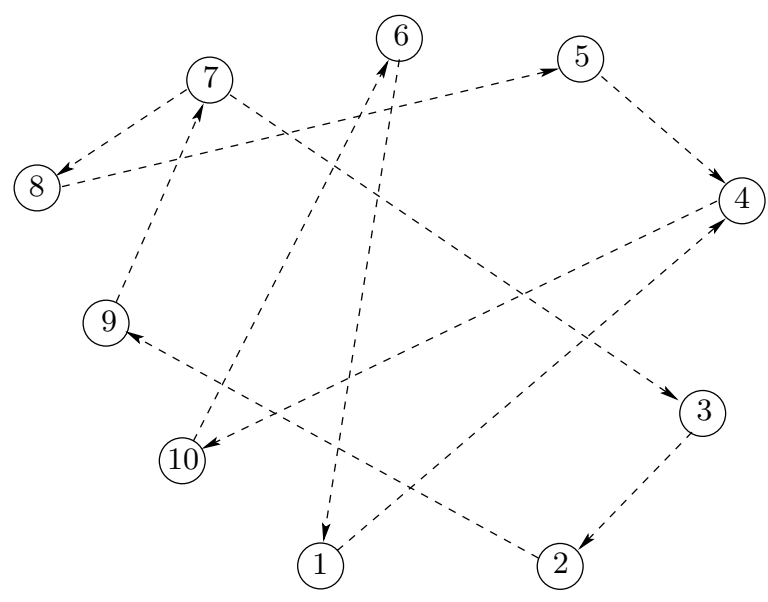

Fig. 1. Communication graph structure
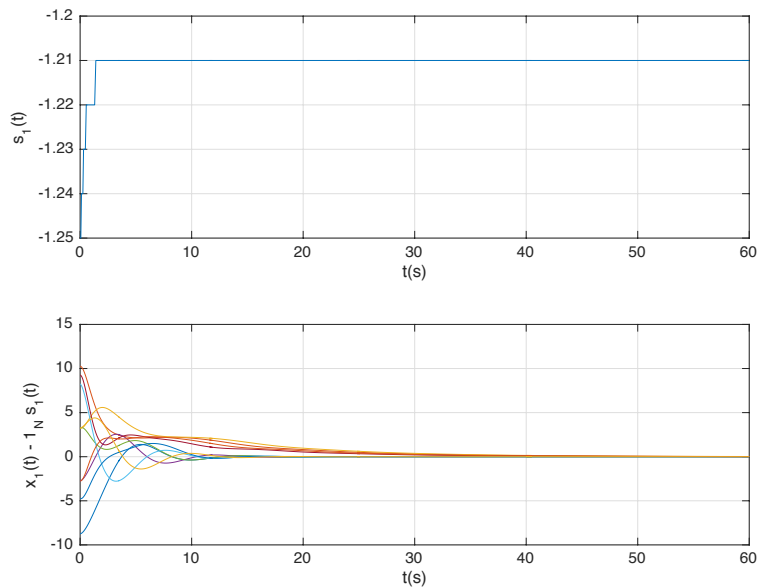

Fig. 2. Perfect measurements: System average and synchronization error evolution

\section{B. Case of unreliable measurements}

For the case when there is a non-zero bias in the measurements of inter-agent positions, $x_{1 i}-x_{1 j}$ we consider two scenarios. In one, we apply the correction term $v(t)$
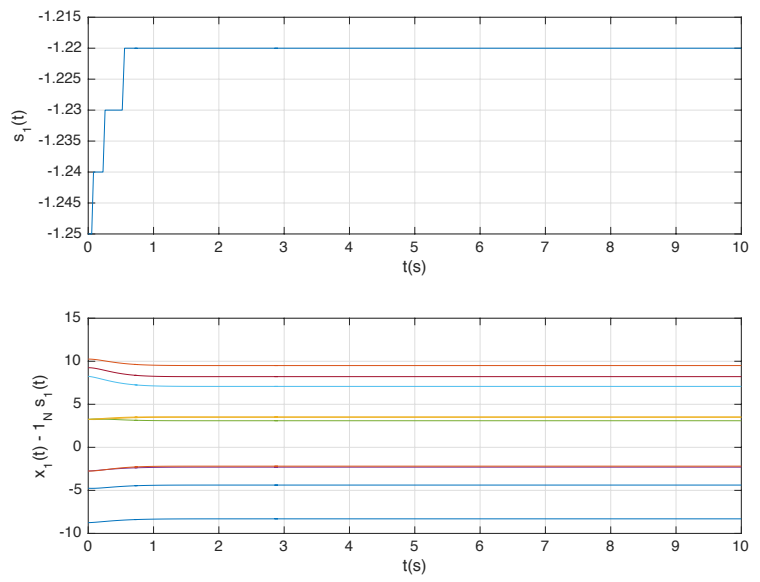

Fig. 3. With bias: System average and synchronization error evolution 
as prescribed by (42) and in the second variant, we apply no correction, i.e. $v(t)=0$. For the bias correction implementation, we choose, $\alpha=0.5$ while $\gamma, \sigma$ are selected as per requirements of Proposition 3. The bias are chosen from uniformly distributed random variables with unity bound on each component of $\delta$.

Figures 3-4 display the simulation results when the correction term $v(t)$ is applied to the control law. As expected by theory, the average value, $s_{1}(t)$ and synchronization errors $\eta_{1}(t)$ converge to constant values, but consensus is not achieved in the presence of bias. The velocity states, $x_{2}(t)$ however converge to zero as expected.

In the absence of a correction term in the control, figure 5 indicates that the average values diverge. The results presented agree well with the theoretical findings presented in earlier sections. The correction term leads to slightly larger synchronisation error in lieu of which we obtain bounded state trajectories in presence of measurement bias.
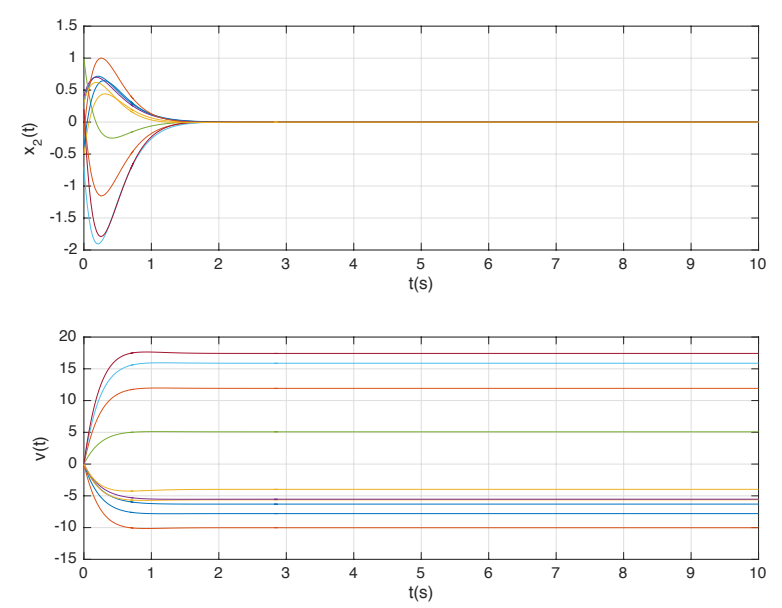

Fig. 4. With bias: $x_{2}(t)$ and bias correction $v(t)$ evolution
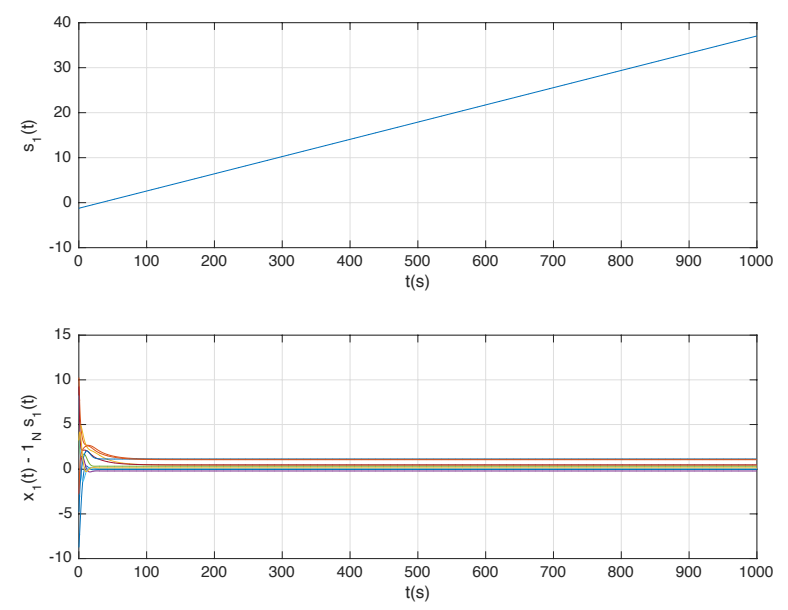

Fig. 5. With bias: System average and synchronization error with correction term, $v(t)=0$

\section{Conclusions}

We have presented some preliminary, but original, results on synchronization of double-integrator systems interconnected over directed graphs and under measurement bias. We have established consensus in a practical sense. That is, we showed that all systems reach a set-point that is in a neighbourhood of the ideal consensus state (that is in the case of reliable measurements). Our results, which are established via Lyapunov's direct method, constitute are a first step into the analysis and design of second-order networked systems over directed graphs.

\section{Acknowledgments}

This work was partially carried out while A. Loria was a visiting professor at the Systems and Control Group of the Indian Institute of Technology, under the sponsorhip of this institution. Additionally, this work was supported by Univ Paris Saclay (grant no. 17IDEXCONSYNHE) and by the goverment of the Russian Federation (Grant 08-08).

\section{REFERENCES}

[1] W. Ren and R. W. Beard, "Consensus algorithms for double-integrator dynamics," Distributed Consensus in Multi-vehicle Cooperative Control: Theory and Applications, pp. 77-104, 2008.

[2] N. R. Chowdhury, S. Sukumar, and N. Balachandran, "Persistence based convergence rate analysis of consensus protocols for dynamic graph networks," European Journal of Control, vol. 29, pp. 33-43, 2016.

[3] M. Maghenem and A. Loría, "Lyapunov functions for persistentlyexcited cascaded time-varying systems: Application to consensus," IEEE Transactions on Automatic Control, vol. 62, pp. 3416-3422, July 2017.

[4] N. R. Chowdhury, S. Sukumar, M. Maghenem, and A. Loría, "On the estimation of the consensus rate of convergence in graphs with persistent interconnections," International Journal of Control, vol. 91, no. 1, pp. 132-144, 2018.

[5] E. Panteley and A. Loría, "Synchronization and dynamic consensus of heterogeneous networked systems," IEEE Transactions on Automatic Control, vol. 62, pp. 3758-3773, Aug 2017.

[6] J. Thienel and R. M. Sanner, "A coupled nonlinear spacecraft attitude controller and observer with an unknown constant gyro bias and gyro noise," IEEE Transactions on Automatic Control, vol. 48, pp. 20112015, Nov 2003.

[7] J. D. Boskovic, S.-M. Li, and R. K. Mehra, "A globally stable scheme for spacecraft control in the presence of sensor bias," in Aerospace Conference Proceedings, 2000 IEEE, vol. 3, pp. 505-511 vol.3, 2000.

[8] N. Metni, J. M. Pflimlin, T. Hamel, and P. Soueres, "Attitude and gyro bias estimation for a flying uav," in 2005 IEEE/RSJ International Conference on Intelligent Robots and Systems, pp. 1114-1120, Aug 2005.

[9] R. Mahony, T. Hamel, and J. M. Pflimlin, "Nonlinear complementary filters on the special orthogonal group," IEEE Transactions on Automatic Control, vol. 53, pp. 1203-1218, June 2008.

[10] P. Patre and S. M. Joshi, "Accommodating sensor bias in MRAC for state tracking," in AIAA Guidance, Navigation, and Control Conference, p. 6605, 2011.

[11] W. Ren, R. W. Beard, and T. W. McLain, Coordination variables and consensus building in multiple vehicle systems, vol. 309 of Lecture Notes in Control and Information Sciences, ch. in Cooperative Control, pp. 171-188. V. Kumar, N. E. Leonard, and A. S. Morse, eds., Berlin: Springer-Verlag, 2005.

[12] J. M. Ortega, Matrix theory: A second course. Springer Science \& Business Media, 2013. 